\title{
Fast neutron irradiation long term effect on Ocimum basilicum germination and initial vegetative parameters
}

\author{
Erika Kurucz ${ }^{1}$ - Ákos Keserü ${ }^{1}$ - Gabriella Antal ${ }^{1 *}$ \\ ${ }^{1}$ University of Debrecen, Faculty of the Agricultural and Food Sciences and Environment Management, Institute of Horticulture, \\ 138. Böszörményi str., Debrecen, H-4032, Hungary \\ *Correspondence: antal.gabriella@agr.unideb.hu
}

\begin{abstract}
SUMMARY
The goal of this study is to assess the long-term (3-year) effects of rapid neutron irradiation using an Americium-Beryllium source on two generations (MO and M1) of Ocimum basilicum commercial trait. Seeds of each variety and generation were irradiated with 0, 7.5, 20, 40, and 50 Gray in 2018, and then seeded in 2021 - with their corresponding controls - to see how the different irradiation treatments affected germination and early vegetative metrics three years after irradiation. In the first generation, no significant changes between the treatments were seen. Increased irradiation dosage resulted in a significant decrease in germination \% in the second generation (M1), but a significant increase in seedling height. The MO generation seedlings, on the other hand, were substantially shorter than controls. In the M0 generation, the higher dosages (40 and 50 Gray) were deadly, and seedlings were terminated 7-10 days after germination. The irradiation dose of 20 Gray was shown to be the most successful in inducing viable and beneficial alterations for phenotypic characteristics in basil. When compared to control plants, the lower (7.5 and 20 Gray) bombardment generated leaf anomalies such as smaller leaves and internodes, a split apex, and a ribbon-like stem.
\end{abstract}

Keywords: Ocimum basilicum, fast neutron irradiation; germination percentage, RAPD, ISSR, DNA marker

\section{INTRODUCTION}

Ocimum species are used widely in food products, beverages, medicines, cosmetics as well as in perfumery. The main cultivated varieties belong to $O$. basilicum L., but there are many other cultivars with economic significance, for example, $O$. americanum (hoary basil) and $O$. gratissimum (clove basil). There are about 140 to 160 species in this genus broadly dispersed over the warm regions of the word. They differ in habits, stress tolerance, chemical and aromatic composition. Among different Ocimum species, there are inter, and intraspecific hybrids which are cultivated extensively for essential oil production throughout the world (Chaudhary et al., 2020) (Chaudhary et al., 2020; Dhawan et al., 2016; Lai et al., 2013). The determination and differentiation of all varieties from each other is particularly challenging because of these natural and/or artificial hybridization (Kumar et al., 2016; Pyne et al., 2018).

The classical breeding method (selection and crossing) is the determining method in these species. The wide diversity of the genus, the huge numbers of unstable traits and segregates within the progenies all make the breeding procedures extremely difficult. But at the same time, the wideness of the gene pool offers lots of opportunities which could be beneficial for the breeding programme of new and better cultivars. The main goal of the basil breeding is to gain high content of volatile oil, beneficial composition, resistance against stress and different plant pathogens and fungus diseases, like Cercospora leaf spot (Cercospora ocimicola), Downy mildew (Peronospora belbahrii), Fusarium wilt (Fusarium oxysporum), which cause great yield loss and decries the value of the products.

The application of inducted mutations has been a perspective tool in breeders' hands since the 1970s.
According to the FAO/IAEA Mutant Variety Database, there are 3281 mutant varieties or breeding sources within the cultivated plants. However, to date, there has been no mutation induction based breeding program that resulted in new varieties (FAO/IAEA, 2015; IAEA, 2015). However, the results of the sources that mention inducted mutation of Ocimum L., it was confirmed that induced mutation can be applied to create variation in the levels of antioxidant activity in $O$. basilicum and can therefore serve as a tool for breeding new variety for functional food/feed material (Asante et al., 2016).

Unlike the electrons and protons, neutrons don't ionize the atoms (excite the electron) in the same manner due to their absence of charges. Nevertheless, neutron interactions are broadly ionizing, as it can be observed when a recoiled nucleus causes a number of traditional ionization in other atoms, or when an electron is removed from an atom by photon (gamma ray), due to gamma emission caused by neutron absorption. Neutron radiation is much more penetrating than alpha or beta radiations, even in some cases than gamma radiation, which is impeded in materials of high atomic number. A low energy gamma ray could be more penetrating than a high energy neutron, in the case of materials like hydrogen, because of the low atomic number (Yue et al., 2013).

Instead of the importance of the inducted mutations on the plant breeding we have very little information about the long-term impact of fast neutron irradiation. Only the short-term effect has been considered in most of the paper in connection with inducted mutation. In the case of the irradiated stored seeds, the one-two or three years impact of storage on the mutation has not yet been studied. Most of the limited papers related to the genetic stability of fast neutron mutants have been conducted in the previous decade and only a few of 
these were based on crop plants, and there is not a single one that studies the storage effect on the M0 generation.

When conducting mutation induction treatment for practical breeding purposes, since the sensitivity of the plant species against the mutagen may vary, before any large scale mutagenic treatment, most necessarily, preliminary sensitivity tests must be run to determine the optimal doses for the studied species. The applicability of the determined dosage may differ within the varieties. According to Kazama et al. (2008) in general, "the objective is to use a dosage and rate in which $50 \%$ of the M1 seeds will germinate and produce seed for the next generation".

The primary necessity in plant breeding is the maintenance of genetic stability during the process of adoption of new varieties, germplasm conversation, and transgenic plant production. The molecular basis of the valuable traits, including the genes and pathways have to be elucidated.

The molecular tools for the detection of even one nucleotide change is fairly vide nowadays, like (MutMap) is a forward genetics method devised to accelerate the process of crop breeding and rapid isolation of genes over QTL mapping (Quantitative Trait Loci) or gene cloning. This is considerably expensive and time consuming, because the technique principle behind MutMap is the identification of mutant phenotype by whole genome sequencing the self pollinated M2 or the segregated M3 generation. Instead of expensive and time consuming methods, the inter simple sequence repeat (ISSR), a quick, cost effective and simple technique, which PCR based method that combines most of the advantages of microsatellites (SSRs) and amplified fragment length polymorphism (AFLP) with the universality of random amplified polymorphic DNA (RAPD). It associates the inter microsatellite DNA segment amplification that is present at an amplifiable distance, oriented in opposite direction (Reddy et al., 2002). In the ISSR technique, customarily, 16-25 bp long primers have been used in a single primer PCR reaction targeting multiple genomic loci to amplify mainly the inter-SSR sequences of different sizes. The primers applied in microsatellite assays can be di-nucleotide, trinucleotide, tetra-nucleotide or penta-nucleotide. The primers should be anchored at 3' or 5' end with 1 to 4 degenerate bases extended into the flanking sequences (Zietkiewicz et al., 1994), but they also can be unanchored (Gupta \& Varshney, 2000; Meyer et al., 1993). ISSRs assays are highly reproductive, probably due to the use of longer primers (16-25 mers) as compared to RAPD primers (10- mers), which allows the use of high annealing temperature $\left(45-60{ }^{\circ} \mathrm{C}\right.$ ) leading to higher fidelity. Based on the results dealing with the reproducibility of ISSR profiles, in polyacrylamide gel about $92-95 \%$ of the gained bands could be repeated within the samples of the same varieties and across different PCR runs (Fang \& Roose, 1997). The DNA fingerprint created by ISSR markers are suitable for the characterization of germplasm collections and the investigation of genetic diversity within species (Roy et al., 2015), cultivar identification, (Chowdhury et al., 2002), genomic stability determination within a species or a cultivar. However, they are not entirely suitable for phylogeny comparison at the species level because of their dominant behavior, which is numbers of authors and breeders consideration (Wang et al., 1998; Wang, 2011). However, some studies reported the co-dominant segregations in case of some ISSR markers. Hence it's possible to make the distinction between homozygotes and heterozygotes (Akagi et al., 1996; Wang, 2011; Wu et al., 1994) (Sankar \& Moore, 2001).

The applicability of the selected primer to the distinguish the mutation can be determine by Polymorphic information content (PIC) analysis, which provides an estimation of the discriminating ability of the marker based on the number of noticeable bands and their distribution (Mishra et al., 2015), (Powell et al., 1996). The other expedient indicator for the discrimination potential of any marker system is called Resolving power (RP) (Kumar et al., 2016; Prevost \& Wilkinson, 1999), effective multiplex ratio (EMR), marker index (MI) (Varshney et al., 2007).

Based on the study of Enkhbileg et al. (2019), fast neutron irradiation is highly effective for mutation induction in $O$. basilicum seeds. The higher dosage caused phenotypically detectable deformation/mutation in the M0 generation. Only the 60 Gray fast neutron dosage inhibits the plant development after the germination at $100 \%$. The 50 Gray has also damaged the plant development, but some mutant plants were able to grow and develop, although the generative biology was damaged, and very few seeds develops and other phenotypic polymorphism also were detected within the mutant population like deformed congestion, leaf mutations, spearmint/spiky and split leaves, low growth, tissue chimeras and flower anthocyanins.

In case of this experiment, we studied the fast neutron irradiation three year effect on Ocimum basilicum L. species with different doses and generation, to monitor the spontaneous mutations stability by the time and determine the plant's initial development. In this regard, we have evaluated fingerprinting profiles of the developed mutant regenerants and the original Ocimum basilicum variety using ISSR and RAPD markers to confirm the genetical stability of the plantlets and to compare the discrimination potential of two PCR method.

\section{MATERIALS AND METHODS}

\section{Fast neutron irradiation}

The irradiation treatment was performed at MTA Atomki (Bem tér 18/c, H-4026 Debrecen, Hungary). on 29.03.2018 in cyclotron application laboratory.

The high-intensity neutron irradiation facility (Fenyvesi, 2004. ) at the MGC-20E cyclotron was used. Fast neutrons with broad energy spectrum in the En $=0$ - $18 \mathrm{MeV}$ neutron energy range were produced by bombarding a beryllium target with $\mathrm{Ep}=16.65 \mathrm{MeV}$ protons. For nuclear physics reasons, the irradiation 
field of the neutrons was accompanied by a field of gamma photons. A twin ionization chamber technique (Broerse et al., 1981) was used for monitoring the separate neutron and gamma dose rates. The dosimetry calculations for the seeds were based on the recommendations of the International Atomic Energy Agency (IAEA, 1967). The material of the seeds was modeled by a medium that contains equal number of hydrogen and carbon atoms. The absorbed neutron (Dn) and gamma (Dg) doses were calculated for this medium. The contribution of the gamma dose to the total dose (Dtot) was Dg/Dtot $=\mathrm{Dg} /(\mathrm{Dn}+\mathrm{Dg})=(14.3)$ $\%$ for each irradiated seed sample. It is important to emphasize that the biological effectiveness of $1 \mathrm{~Gy}$ neutron dose is 2-11 times higher than that of $1 \mathrm{~Gy}$ gamma dose depending on the neutron energy.

\section{Sowing of irradiated seeds and nursery operations}

Two generation were used in this study, M0, which are the irradiated seeds, M1 the first open pollinated generation. Every generation of control, 7.5 Gray, 20 Gray, 40 Gray, 50 Gray dose 20 surface sterilized seeds were sown in Petri-dish (10 cm diameter, polystyrene, VWR International Ltd., Hungary) in four replicates, into white filter paper (VWR International Ltd., Hungary) and were soaked with $3 \mathrm{ml} \mathrm{RO}$ water and 3 $\mathrm{ml}$ of $\mathrm{RO}$ water was poured onto the filter paper at one time during germination assay. The seeds were germinated under 12 hours supplemental light (HortiLED Multi 4DIM, Hortilux, Netherlands) with $230 \mu \mathrm{mol} \mathrm{m} \mathrm{m}^{-2} \mathrm{~s}^{-1}$ PPFD (photosynthetic photon flux density) light intensity.

The experiments were carried out at University of Debrecen, Faculty of the Agricultural and Food Sciences and Environment Management, in Biological
Research and Plant Experiment Greenhouse (Biodrome) from March 2021 to June 2021 (Hungary), during natural long-day conditions. The airtemperature computer controlled (Priva Climate Computer) was at least $20.5{ }^{\circ} \mathrm{C}$ at daytimes and at least $16^{\circ} \mathrm{C}$ at nights during our experiment.

\section{DNA isolation}

Young shoots $(15-20 \mathrm{~cm})$ were collected from phenotypically selected 6-month-old fast neutron irradiated (7.5 Gy, $20 \mathrm{~Gy}, 40 \mathrm{~Gy}, 50 \mathrm{~Gy}$ ) mutants of Ocimum basilicum $\mathrm{L}$. The nodal segments with axillary and apical buds were separated and ground with liquid nitrogen, in order to prepare a homogenized sample. Total genomic DNA was isolated from the prepared plant sample using the ZR Plant/Seed DNA (Zymo Research Co.) kit and its protocol described by the manufacturer. The quantity of isolated genomic DNA samples was quantified spectrophotometrically (Thermo Scientific NanoDrop 2000 full-spectrum, UVVis spectrophotometer) by measuring absorbance at $260 \mathrm{~nm}$ and $280 \mathrm{~nm}$ for the OD260/OD280 ratio. Also, the quality was checked by visualizing it under UV light after electrophoresis on $0.8 \%$ agarose gel. The stock DNA was diluted to make a required working solution of $30 \mathrm{ng} / \mu \mathrm{l}$.

\section{PCR amplification}

Amplification was performed in $25 \mu \mathrm{L}$ using PCR mixture of consisting of $23 \mu \mathrm{L}$ DreamTaqGreen Mastermix (which contains DreamTaqgreen DNA polymerase, dNTP (dATP: dTTP: dCTP: dGTP in 1:1:1:1 parts) and DNA polymerase buffer with $\mathrm{MgCl} 2), 1 \mu \mathrm{L}$ genomic DNA and $1 \mu \mathrm{L}$ ISSR primer (Table 1).

Table 1. The primer and PCR details used to $O$. basilicum DNA assay

\begin{tabular}{|c|c|c|c|c|c|c|}
\hline Primer & $\begin{array}{c}\text { Sequence } \\
5 \text { '-3' }\end{array}$ & $\begin{array}{l}\text { Melting temp. } \\
\left({ }^{\circ} \mathrm{C}\right)\end{array}$ & $\begin{array}{c}\text { Number of } \\
\text { cycles }\end{array}$ & $\begin{array}{c}\text { Denat. temp } \\
\left({ }^{\circ} \mathrm{C}\right)\end{array}$ & Program & Ending \\
\hline UBC-818 & CA CA CA CA CA CA CA CAG & 52 & 30 & \multirow{10}{*}{$\begin{array}{l}94{ }^{\circ} \mathrm{C}- \\
1 \mathrm{~min} .\end{array}$} & \multirow{10}{*}{$\begin{array}{c}94^{\circ} \mathrm{C}- \\
20 \mathrm{sec} . \\
\text { a.h-20 sec. } \\
72{ }^{\circ} \mathrm{C}- \\
2 \mathrm{~min} .\end{array}$} & \multirow{10}{*}{$\begin{array}{l}72^{\circ} \mathrm{C}- \\
6 \mathrm{~min} .\end{array}$} \\
\hline UBC-836 & AGA GAG AGA GAG AGA GYC & 50.2 & 30 & & & \\
\hline UBC-841 & GAG AGA GAG AGA GAG AYC & 48.5 & 30 & & & \\
\hline UBC-856 & ACA CAC ACA CAC ACA CYA & 52.8 & 30 & & & \\
\hline OPZ-09 & CACCCCAGTC & 35.8 & 40 & & & \\
\hline OPI-06 & AAGGCGGCAG & 41.1 & 40 & & & \\
\hline OPI-07 & CAGCGACAAG & 33.5 & 40 & & & \\
\hline OPI-08 & TTTGCCCGGT & 32.6 & 40 & & & \\
\hline OPI-09 & TGGAGAGCAG & 33.1 & 40 & & & \\
\hline OPI-10 & ACAACGCGAG & 33.2 & 40 & & & \\
\hline
\end{tabular}

The PCR conditions were carried out using a thermal cycler (MJ Research PTC-150 Thermal Cycler) using the parameters above in Table 1. Amplicons were electrophoresed on $1.5 \%$ agarose gel and stained with EcoSafe $(5 \mu \mathrm{L} / \mathrm{gel})$. 100bp plus DNA Ladder (ThermoFisher Scientific Co.) was used as the DNA marker. and bands were visualized under UV light and photographed using the Gel Documentation equipment.
Amplified ISSR products were analyzed with a software called PhylElph. The binary matrixes were scored for band presence or absence for each accession (presence $=1$. absence $=0$ ). and a binary data matrix was formed. Primer banding characteristics such as the number of total bands (TB). the number of polymorphic bands (PB). and the percentage of polymorphic bands (PPB) were acquired. 
To check the genetic relation among the Ocimum basilicum and its mutants. data matrix of ISSR markers was converted into genetic similarity matrix using Jaccard coefficient (Jaccard. 1908) in SPSS 17.0 (SPSS Inc.).

The performance of the markers was measured in order to analyze the suitability of ISSR and RAPD markers to determine the genetic profiles of Ocimum basilicum mutants. using three parameters:

polymorphic information content (PIC)

effective multiplex ratio (EMR) marker index (MI)

The PIC value for each locus was calculated as proposed by Roldan-Ruiz et al. (2000). PICi $=2 \mathrm{fi} *(1$ fi); where PICi is the PIC of the locus i. fi is the frequency of the amplified fragments (band present). and (1 - fi) is the frequency of non-amplified fragments (band absent). The frequency was calculated as the ratio between the number of amplified bands at each locus and the total number of accessions (excluding missing data). The PIC of each primer was calculated using the average PIC value from all loci of each primer.

The marker index (MI) was calculated as described by Varshney et al. (2007). MI = EMR * PIC; where EMR is the product of the fraction of polymorphic loci and the number of polymorphic loci for an individual assay.

The resolving power (RP) of each primer was calculated according to Prevost and Wilkinson (1999). $\mathrm{RP}=\sum \mathrm{Ib}$; where $\mathrm{Ib}$ represents the informative fragments. The Ib can be represented on a scale of $0-1$ by the following formula: $\mathrm{Ib}=1-[2 *(0.5-\mathrm{p})]$; where $\mathrm{p}$ is the proportion of cultivars containing the band lished results.

\section{RESULTS AND DISCUSSION}

The germination percentage and the seedlings length of the irradiated generation

The germination percentage in the first generation did not show any significant differences compared to the control (Figure 1). In the 40 Gray M1 generation were the least germinated seeds were detected. In the case of the first generation. the seedlings were significantly shorter than the control. regardless of the bombardment dosage. The higher dose of irradiation caused early seedling necrosis and abortion after 7-10 days after germination (Figure 1-2). Based on our previous germination assays. even the highest dose did not inhibit significantly the germination process. However. at a higher dosage. the seedlings' development stopped in cotyledons. no complete plants developed from 60GY doses (Enkhbileg et al.. 2019). After three years of storage. the germination percentage was lower in all cases. so the initial seedlings development performance shows the mutation presence in the genome.

In the case of the 7-day old seedlings' length. the effect of radiation was markedly visible in the first generation. Within the open-pollinated second generations. rather some moderate positive effect was observed. This tendency was also visible in the biomass production of the second generation (Figure 2).

Figure 1. The germination percentage of $\boldsymbol{O}$. basilicum mutant plant

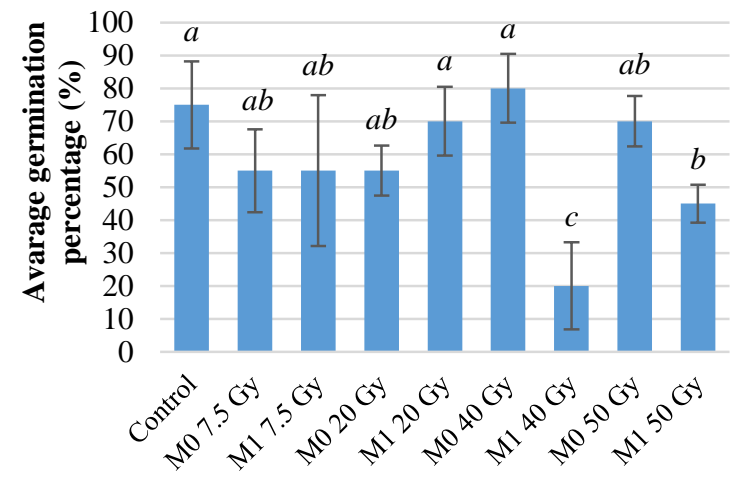

Notes: M0 is the irradiated seeds. M1 the first open pollinated generations. University of Debrecen. Biological Research and Plant Experiment Greenhouse - Biodrome. Debrecen. Hungary). The different letters at the top of the column represent the significant differences. which were determined with Dunnett's test at a probability level of $5 \%$.

Figure 2. The seedlings length of mutant $\boldsymbol{O}$. basilicum mutant plant

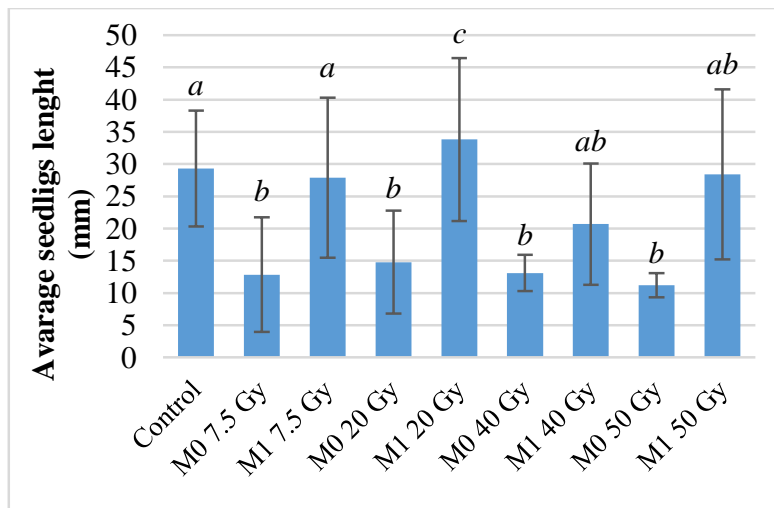

Notes: University of Debrecen. Biological Research and Plant Experiment Greenhouse - Biodrome. Debrecen. Hungary. The different letters at the top of the column represent the significant differences. which were determined with Dunnett's test at a probability level of $5 \%$.

\section{The vegetative parameters of irradiated basil generations}

The lower irradiation dosage did not cause a significant effect on the height of plants and the number of branches. The higher dose markedly inhibits the initial growth in the case of 40 and 50 Grays treatments in the first irradiated generation couldn't develop after germination. the 20 Gray treatment caused a $25-50 \%$ height decrease and resulted in 2-3 branches louse. In the first year (2018) of the fast neutron irradiation. the initial growth was less hampered (Figure 3-4). 
Figure 3. The plant height of $\boldsymbol{O}$. basilicum mutant plant on four different time
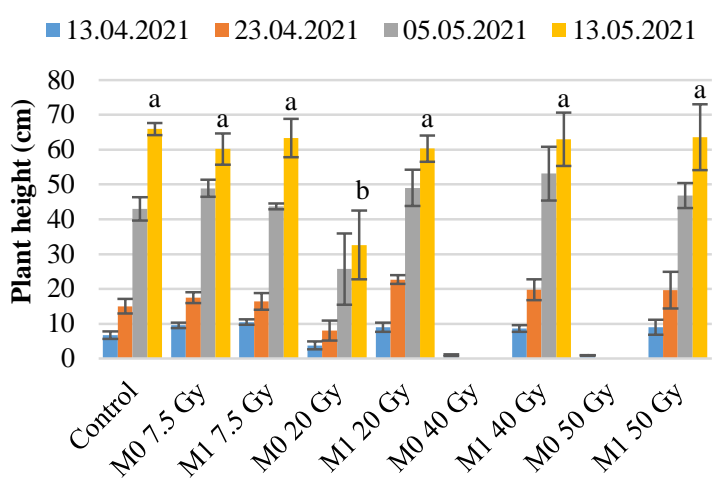

Notes: University of Debrecen. Biological Research and Plant Experiment Greenhouse - Biodrome. Debrecen. Hungary. The different letters at the top of the column represent the significant differences. which were determined with Dunnett's test at a probability level of $5 \%$.

Figure 4. The number of branches of $\boldsymbol{O}$. basilicum mutant plant

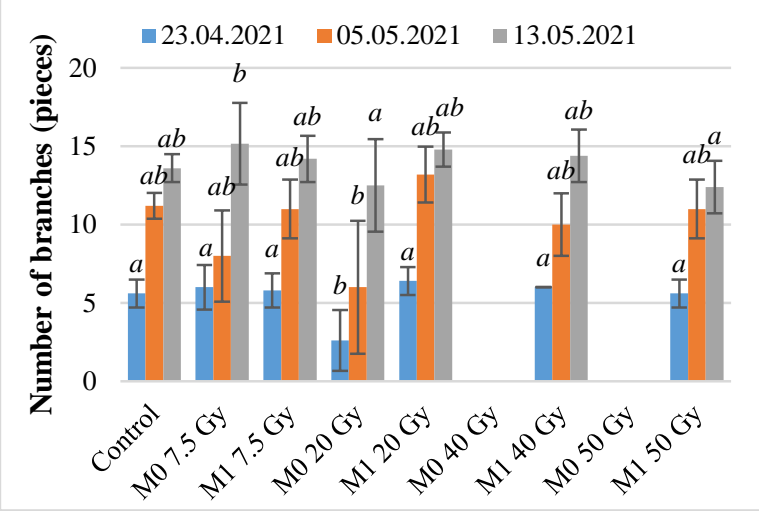

Notes: University of Debrecen. Biological Research and Plant Experiment Greenhouse - Biodrome. Debrecen. Hungary. The different letters at the top of the column represent the significant differences. which were determined with Dunnett's test at a probability level of $5 \%$.

The length of internode in the first generation and second generations were significantly different. It can be said generally. in the first generation the internode length was significantly lower. and in the case of the second generation was also visible some moderate decrease. though this phenomenon was not typically in the biomass production. It could be mean. that the mutant plant could be able to accumulate more biomass or moisture. or could grow more branches or leaves. As Figure 4 was shown. the differences in the number of branches between the treatment was not significant. but the dynamic of the growth was excursive (Figure 5-6). In terms of the number of flowers. it should be said. that the higher dosage. which had resulted in fully developed plants. reduced the number of flowers in the first generation.
In the case of the internode length. the irradiation had some kind of effect in all treatments and generations. In the first generation. the internode reduction was detected from 117.2 to $40 \mathrm{~mm}$ (Figure $5)$. In the second generation. the otherwise tendency was observed. The biomass production of 20 Gray treatment reduced in the first generation and the lower dosage moderate positive effect also was observed (Figure 6).

Figure 5. The internodes length of $\boldsymbol{O}$. basilicum mutant plant

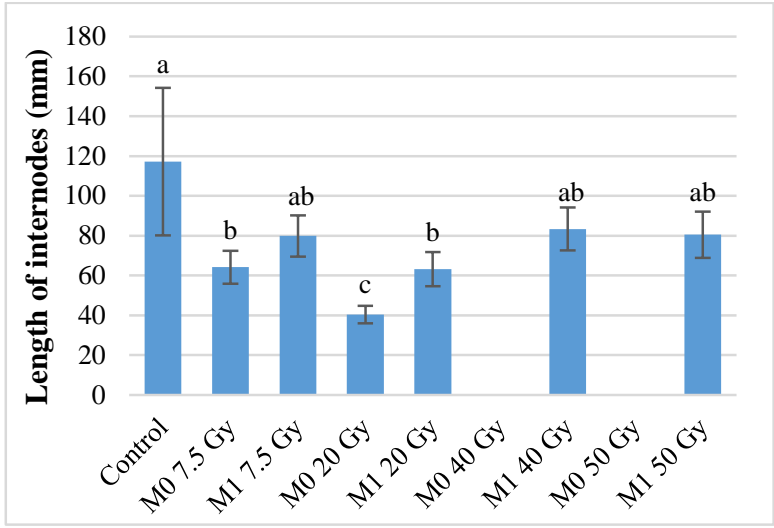

Notes: University of Debrecen. Biological Research and Plant Experiment Greenhouse - Biodrome. Debrecen. Hungary. The different letters at the top of the column represent the significant differences. which were determined with Dunnett's test at a probability level of $5 \%$.

Figure 6. The fresh biomass production of $\boldsymbol{O}$. basilicum mutant plant

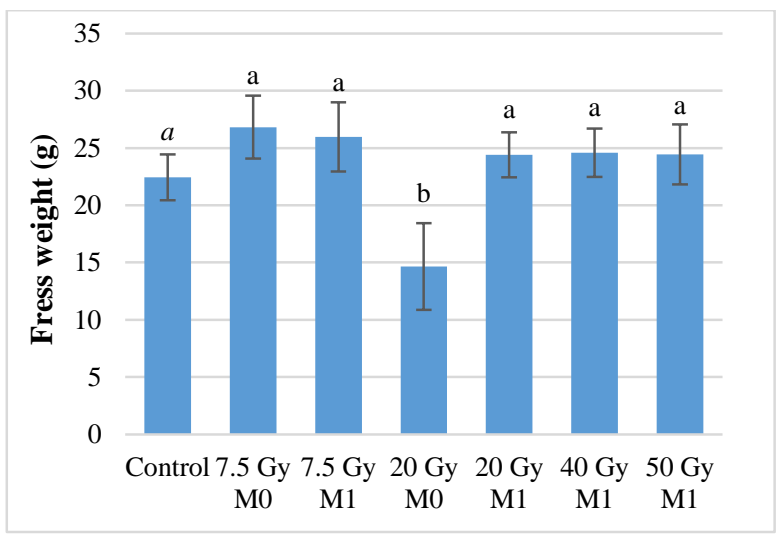

Notes: University of Debrecen. Biological Research and Plant Experiment Greenhouse - Biodrome. Debrecen. Hungary. The different letters at the top of the column represent the significant differences. which were determined with Dunnett's test at a probability level of $5 \%$.

Stoma density. only the abaxial stomas' number were significant higher within the control plants. The shape of the stomata (length and width) were similar in all treatment and generation (Figure 7-8). 
Figure 7. The stoma density of $\boldsymbol{O}$. basilicum mutant plant

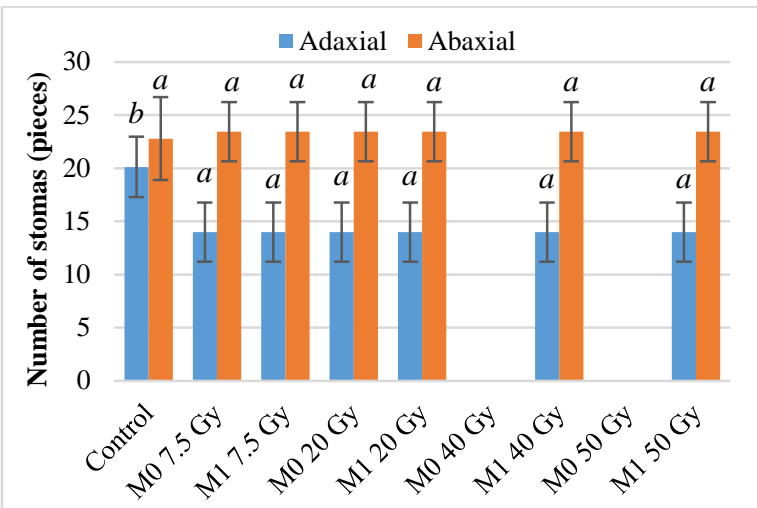

Notes: University of Debrecen. Biological Research and Plant Experiment Greenhouse - Biodrome. Debrecen. Hungary. The different letters at the top of the column represent the significant differences. which were determined with Dunnett's test at a probability level of $5 \%$.

Figure 8. The stoma appearances of $O$. basilicum mutant plant

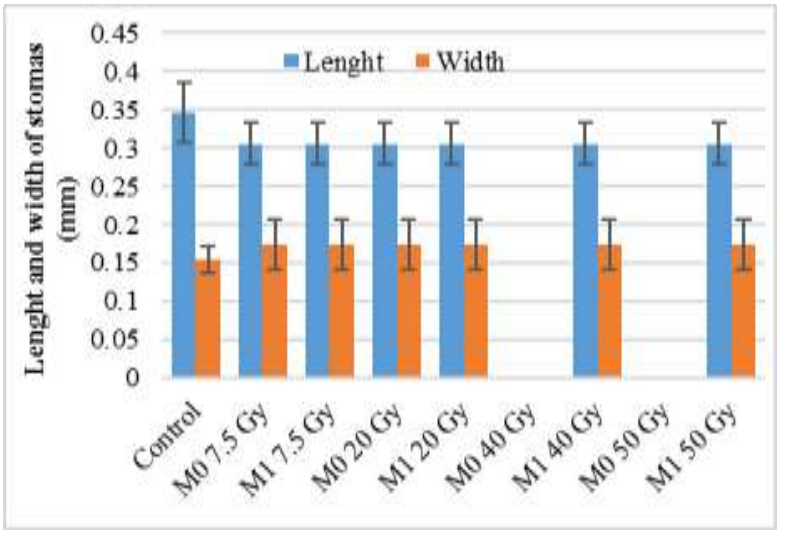

Notes: University of Debrecen. Biological Research and Plant Experiment Greenhouse - Biodrome. Debrecen. Hungary. No significant differences. which were determined with Dunnett's test at a probability level of $5 \%$.

\section{Performance of DNA markers}

In order to boost the polymorphism and specificity. all the used dinucleotide primers were anchored at their 3 '-end with one or two degenerate nucleotide(s). The four ISSR primers and six RAPD primers used in this study yielded 79 loci ranging from 0.4 to $1.7 \mathrm{~kb}$. out of which 47 loci were polymorphic in nature representing $59 \%$ polymorphism. In the case of the 2018 experiment the ISSR loci were more than $95 \%$ polymorphic. The number of loci reproduced by these arbitrary primers was found to range from 4 to 14 of different sizes with an average frequency of 16.43 loci per ISSR primer (Table 2).

Based on the genetic distance (Table 3). there are no stronger genetic relationships within the same dosage. mainly in the lower dosage. Interestingly. the mutant in higher dosage treatment (40-50 Gray) showed lower genetic (0-12.5) distance in al studied generation compared to the lower dosage treated mutant (36.876.47). Since these mutation will be detectable in the M1 to M4 generations. in the next generation we are going to determine the genetic stability of the mutation.

More then the $50 \%$ of ISSR loci were polymorphic at species level for each primer except UBC 818. UBC 841 (Table 3). The RAPD loci were less polymorphic. In this study. the highest PIC value of 0.48 was observed for primer UBC 818. and the lowest PIC value of 0.02 was observed for primer OPI-09. with an overall average PIC value of 0.344 per primer (Figure 9).

Table 2. The discriminative potential of the ISSR and RAPD primers

\begin{tabular}{ccccccc}
\hline & $\begin{array}{c}\text { Total bands } \\
(\text { TB })\end{array}$ & Polimorphic loci (PB) & P\% & PIC & EMR & MI \\
\hline UBC-818 & 8 & 8 & 100.0 & 0.48 & 3.50 & 1.66 \\
UBC-836 & 8 & 4 & 50.0 & 0.38 & 3.38 & 1.27 \\
UBC-841 & 6 & 3 & 50.0 & 0.35 & 3.50 & 1.21 \\
UBC-856 & 13 & 7 & 53.8 & 0.42 & 3.65 & 1.42 \\
OPZ-09 & 5 & 3 & 60.0 & 0.35 & 3.11 & 1.08 \\
OPI-06 & 4 & 1 & 25.0 & 0.05 & 2.19 & 0.11 \\
OPI-07 & 14 & 12 & 85.7 & 0.35 & 4.41 & 1.54 \\
OPI-08 & 11 & 5 & 45.5 & 0.17 & 3.12 & 0.54 \\
OPI-09 & 10 & 2 & 20.0 & 0.02 & 3.20 & 0.07 \\
OPI-10 & 5 & 2 & 40.0 & 0.11 & 2.93 & 0.32 \\
\hline
\end{tabular}


Table 3. The genetical distance of the mutants based on the UBC-856 primers' loci

\begin{tabular}{cccccccccc}
\hline & Control & M0 7.5 Gy & M1 7.5 Gy & M0 20 Gy & M1 20 Gy & M0 40 Gy & M1 40 Gy & M0 50 Gy & M1 50 Gy \\
\hline Control & 0.00 & 36.84 & 64.71 & 66.67 & 76.47 & 62.50 & 75.00 & 62.50 & 62.50 \\
M0 7.5 Gy & 36.84 & 0.00 & 40.00 & 52.38 & 50.00 & 47.37 & 47.37 & 47.37 & 47.37 \\
M1 7.5 Gy & 64.71 & 40.00 & 0.00 & 26.32 & 11.11 & 17.65 & 5.88 & 17.65 & 17.65 \\
M0 20 Gy & 66.67 & 52.38 & 26.32 & 0.00 & 15.79 & 33.33 & 22.22 & 33.33 & 33.33 \\
M1 20 Gy & 76.47 & 50.00 & 11.11 & 15.79 & 0.00 & 17.65 & 5.88 & 17.65 & 17.65 \\
M0 40 Gy & 62.50 & 47.37 & 17.65 & 33.33 & 17.65 & 0.00 & 12.50 & 0.00 & 0.00 \\
M1 40 Gy & 75.00 & 47.37 & 5.88 & 22.22 & 5.88 & 12.50 & 0.00 & 12.50 & 12.50 \\
M0 50 Gy & 62.50 & 47.37 & 17.65 & 33.33 & 17.65 & 0.00 & 12.50 & 0.00 & 0.00 \\
M1 50 Gy & 62.50 & 47.37 & 17.65 & 33.33 & 17.65 & 0.00 & 12.50 & 0.00 & 0.00 \\
\hline
\end{tabular}

Figure 9. Agarose gel picture of OPI-08 (A). and UBC-856 (C). the phylogenetic tree. by UPMGA method (B: OPI-09-C: UBC-856)

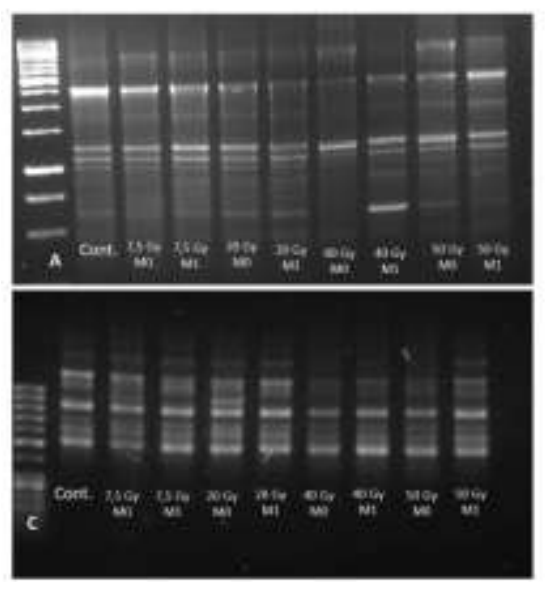

\section{CONCLUSIONS}

As the previous study concluded (Enkhbileg et al., 2019). in 2018 fast neutron irradiation is highly effective for mutation induction in the case of Ocimum basilicum seeds. The higher dosage caused phenotypically detectable deformation/mutation in the M0 generation in the first year of the irradiation. However. in the next generations. the mutation should be suppressed by the DNA correction and rearrangement process. as the Ocimum basilicum is a polyploid species. So many mutations. during the storage period or the selection through the generation. should be covered. In the first year of the irradiation. the 60 Gray fast neutron dosage inhibits the plant development after the germination and the 50 Gray has also damaged the plant development. but we were able to grow some mutant plants from it. In contrast. in the third year. even lower dosages (40-50 Gray) were lethal and caused seedlings abortion. We detected other phenotypic polymorphisms within the mutant population like deformed congestion. leaf mutations. spearmint/spiky and split leaves. low growth. tissue chimeras. and flower anthocyanin in the first year. but in the third year. the visible mutations were fewer and more restrained.

In the case of the initial growth. the seedling height or the internode length should be a good marker. to

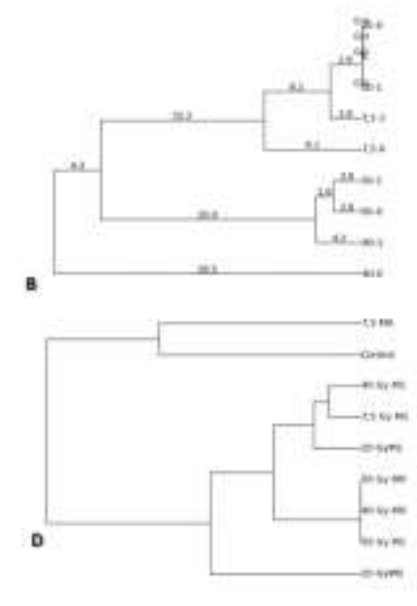

largely determine the genetic variability and valuable mutant selection in an early stage of the breeding program.

The modern molecular biology assay is too expensive and time-consuming for the practical company and breeders. Based on our study. the ISSR and RAPD are cheap. easy. and fast methods. which could be beneficial for marker-assisted breeding of polyploid or species with unknown genomes. Our analysis clearly shows that ISSR markers are more robust compared to RAPD markers. means for creating fingerprinting keys. and have the potential to identify mutation-specific markers for Ocimum basilicum. The modern technologies provide an easy and fast opportunity for the generation of fingerprinting keys. the identification of species and cultivar-specific ISSR or RAPD markers. and the development of function or phenotype specific markers in order to serve and modern marker-assisted selection method for the basil varieties and species.

\section{ACKNOWLEDGEMENTS}

The work/publication is supported by the EFOP3.6.1-16-2016-00022 project. The project is cofinanced by the European Union and the European Social Fund. 


\section{REFERENCES}

Akagi. H.-Yokozeki. Y.-Inagaki. A.-Nakamura. A.-Fujimura. T. (1996): A co-dominant DNA marker closely linked to the rice nuc-lear restorer gene. Rf-1. identified with inter-SSR fingerprinting. Genome Biol Evol. 39. 1205-1209. https://doi.org/https://doi.org/10.1139/g96-152

Asante. I.K.-Annan. K.-Essilfie. M.K.-Tater. V. (2016): Effect of Induced Mutation on Antioxidant Activity in Ocimum basilicum Linn. Natural Science. 8(04). 192 https://doi.org/https://doi.org/10.4236/ns.2016.84022

Broerse. J.J.-Mijnheer. B.J.-Williams. J.R. (1981): European protocol neutron dosimetry for external beam therapy. British Journal of Radiology. 54. 882-898.

Chaudhary. A.-Sharma. S.-Mittal. A.-Gupta. S.-Dua. A. (2020): Phytochemical and antioxidant profiling of Ocimum sanctum. Journal of Food Science and technology. 57(10). 3852-3863.

Chowdhury. M.A.-Vandenberg. B.-Warkentin. T. (2002): Cultivar identification and genetic relationship among selected breeding lines and cultivars in chickpea (Cicer arietinum L.) [journal article]. Euphytica. 127(3). 317-325. https://doi.org/https://doi.org/10.1023/a:1020366819075

Dhawan. S.S.-Shukla. P.-Gupta. P.-Lal. R. (2016): A cold-tolerant evergreen interspecific hybrid of Ocimum kilimandscharicum and Ocimum basilicum: analyzing trichomes and molecular variations. Protoplasma. 253(3). 845-855.

Enkhbileg. E.-Fenyvesi. A.-Bíró. B.-Fári. M.-Kurucz. E. (2019): Mutation induction in sweet basil (Ocimum basilicum L.) by fast neutron irradiation. International Journal of Horticultural Science. 25(1-2). 30-38.

Fang. D.Q.-Roose. M.L. (1997): Identification of Closely Related Citrus Cultivars with Inter-Simple Sequence Repeats Markers. Theoretical and Applied Genetics.. 95. 408-417. https://doi.org/http://dx.doi.org/10.1007/s001220050577

FAO/IAEA. (2015): Protocol for X-ray mutagenesis of plant material: seed. The Plant Breeding and Genetics Laboratory (PBGL) of the FAO/IAEA. Manual. 1-13.

Fenyvesi. A. (2004): Neutron sources for basic and applied research at the MGC-20E cyclotron of ATOMKI. Neutron Measurements and Evaluations for Applications. Budapest. Hungary.

Gupta. P.K.-Varshney. R. (2000): The development and use of microsatellite markers for genetic analysis and plant breeding with emphasis on bread wheat. Euphytica. 113(3). 163-185. https://doi.org/https://doi.org/10.1023/A:1003910819967

IAEA. (1967): International Atomic Energy Agency: Recommendations. Neutron Irradiation of Seeds. Technical Report Series. 76. 74-86. Coordinated Research Activities. 124. (2015).

Jaccard. P. (1908): Nouvelles recherches sur la distribution florale. Bull Soc Vaudoise Sci Nat. 44. 223-270.

Kazama. Y.-Saito. H.-Yamamoto. Y.Y.-Hayashi. Y.-Ichida. H.Ryuto. H.-Fukunishi. N.-Abe. T. (2008): LET-dependent effects of heavy-ion beam irradiation in Arabidopsis thaliana. Plant Biotechnology. 25(1). 113-117.

Kumar. A.-Mishra. P.-Baskaran. K.-Shukla. A.K.-Shasany. A.K.Sundaresan. V. (2016): Higher efficiency of ISSR markers over plastid psbA-trnH region in resolving taxonomical status of genus Ocimum L. Ecology and Evolution. 6(21). 7671-7682. https://doi.org/https://doi.org/10.1002/ece3.2483

Lai. D.-Brötz-Oesterhelt. H.-Müller. W.E.-Wray. V.-Proksch. P (2013): Bioactive polyketides and alkaloids from Penicillium citrinum. a fungal endophyte isolated from Ocimum tenuiflorum. Fitoterapia. 91. 100-106.

Meyer. W.-Mitchell. T.G.-Freedman. E.-Vilgalys. R. (1993): Hybridization probes for conventional DNA fingerprinting used as single primers in the polymerase chain reaction to distinguish strains of Cryptococcus neoformans. Journal of Clinical Microbiology. 31(9). 2274-2280. https://doi.org/https://www.ncbi.nlm.nih.gov/pmc/articles/PMC 265746/

Mishra. P.-Kumar. L.D.-Kumar. A.-Gokul. S.-Ravikumar. K.Shukla. A.K.-Sundaresan. V. (2015): Population Dynamics and Conservation Implications of Decalepis arayalpathra (J. Joseph and V. Chandras.) Venter. a Steno Endemic Species of Western Ghats. India [journal article]. Applied Biochemistry and Biotechnology. 176(5). 1413-1430. https://doi.org/https://doi.org/10.1007/s12010-015-1654-7

Powell. W.-Morgante. M.-Andre. C.-Hanafey. M.-Vogel. J.Tingey. S.-Rafalski. A. (1996): The comparison of RFLP. RAPD. AFLP and SSR (microsatellite) markers for germplasm analysis. Molecular breeding. 2(3). 225-238. https://doi.org/https://doi.org/10.1007/BF00564200

Prevost. A.-Wilkinson. M.J. (1999): A new system of comparing PCR primers applied to ISSR fingerprinting of potato cultivars [journal article]. Theoretical and Applied Genetics. 98(1). 107112. https://doi.org/https://doi.org/10.1007/s001220051046

Pyne. R.M.-Honig. J.A.-Vaiciunas. J.-Wyenandt. C.A.-Simon. J.E. (2018): Population structure. genetic diversity and downy mildew resistance among Ocimum species germplasm. $\begin{array}{llll}\text { BMC plant } & \text { biology. }\end{array}$ https://doi.org/https://doi.org/10.1186/s12870-018-1284-7

Reddy. M.P.-Sarla. N.-Siddiq. E. (2002): Inter simple sequence repeat (ISSR) polymorphism and its application in plant breeding. Euphytica. 128(1). 9-17. https://doi.org/https://doi.org/10.1023/A:1020691618797

Roldan-Ruiz. I.-Dendauw. J.-Van Bockstaele. E.-Depicker. A.-De Loose. M. (2000): AFLP markers reveal high polymorphic rates in ryegrasses (Lolium spp.). Molecular breeding. 6(2). 125-134.

Roy. S.-Banerjee. A.-Mawkhlieng. B.-Misra. A.-Pattanayak. A.Harish. G.-Singh. S.-Ngachan. S.-Bansal. K. (2015): Genetic diversity and population structure in aromatic and quality rice (Oryza sativa L.) landraces from North-Eastern India. PloS one. 10(6). e0129607. https://doi.org/https://doi.org/10.1371/journal.pone.0129607

Sankar. A.A.-Moore. G.A. (2001): Evaluation of inter-simple sequence repeat analysis for mapping in Citrus and extension of the genetic linkage map [journal article]. Theoretical and Applied Genetics. 102(2). 206-214. https://doi.org/https://doi.org/10.1007/s001220051637

Varshney. R.K.-Marcel. T.C.-Ramsay. L.-Russell. J.-Röder. M.S.Stein. N.-Waugh. R.-Langridge. P.-Niks. R.E.-Graner. A. (2007): A high density barley microsatellite consensus map with 775 SSR loci. Theoretical and Applied Genetics. 114(6). 10911103.

Wang. G.-Mahalingan. R.-Knap. H.T. (1998): (C-A) and (G-A) anchored simple sequence repeats (ASSRs) generated poly-morphism in soybean. Glycine $\max$ (L.) Merr. Theor Appl Genet 96. p. 1086-1096. https://doi.org/https://doi.org/10.1007/s001220050843

Wang. X.-m. (2011): Inter-simple sequence repeats (ISSR) molecular fingerprinting markers for authenticating the genuine species of 
rhubarb. Journal of Medicinal Plants Research. 5(5). 758-764. https://doi.org/https://academicjournals.org/journal/JMPR/articl e-full-text-pdf/D9C403718893

Wu. K.-Jones. R.-Dannaeberger. L.-Scolnik. P.A. (1994): Detection of microsatellite polymorphisms without cloning. Nucleic Acids $\quad$ Res 22. 3257-3258 https://doi.org/https://doi.org/10.1093/nar/22.15.3257
Yue. A.-Dewey. M.-Gilliam. D.-Greene. G.-Laptev. A.-Nico. J.Snow. W.M.-Wietfeldt. F. (2013): Improved determination of the neutron lifetime. Physical review letters. 111(22). 222501. https://doi.org/https://doi.org/10.1103/PhysRevLett.111.222501 Zietkiewicz. E.-Rafalski. A.-Labuda. D. (1994): Genome fingerprinting by simple sequence repeat (SSR)-anchored polymerase chain reaction amplification. Genomics. 20(2). 176183. https://doi.org/https://doi.org/10.1006/geno.1994.1151 
